\title{
Citric Acid Crosslinked Carboxymethyl Cellulose-based Composite Hydrogel Films for Drug Delivery
}

\author{
K. K. MALI*, S. C. DHAWALE ${ }^{1}$, R. J. DIAS ${ }^{2}$, N. S. DHANE AND V. S. GHORPADE \\ Department of Pharmaceutics, YSPM'S Yashoda Technical Campus, Faculty of Pharmacy, Wadhephata, Satara-415011, \\ ${ }^{1}$ Department of Pharmacology, School of Pharmacy, SRTM University, Nanded-431 606, ${ }^{2}$ Department of Pharmacy, Government \\ Polytechnic, National Highway No 6, Jalgaon-425 002, India
}

\section{Mali et al.: Carboxymethyl cellulose-based hydrogel films for drug delivery}

\begin{abstract}
The objective of this study was to synthesize and characterize citric acid crosslinked hydrogel films of carboxymethyl cellulose-tamarind gum for topical drug delivery. The hydrogel films were characterized by attenuated total reflectance-Fourier-transform infrared spectroscopy, solid-state ${ }^{13} \mathrm{C}$-nuclear magnetic resonance spectroscopy and differential scanning calorimeter. The prepared hydrogel films were evaluated for the carboxyl content and equilibrium swelling ratio. Moxifloxacin hydrochloride was loaded into these hydrogel films and drug release was monitored in the phosphate buffer pH 7.4. Haemolysis assay was used to study biocompatibility of hydrogel films. Results of the attenuated total reflectance-Fourier-transform infrared spectroscopy, solid-state ${ }^{13} \mathrm{C}$-nuclear magnetic resonance and differential scanning calorimeter confirmed the formation of citric acid-crosslinked hydrogel films. Total carboxyl content of hydrogel film was found to be increased when polymer ratio and amount of citric acid was increased. In contrast, swelling of hydrogel film was found to be decreased with increase in polymer ratio and amount of citric acid. Batch B1 showed highest drug loading with non-Fickian release mechanism. All remaining batches showed nonFickian release behavior with diffusion coefficient greater than 0.5 . Results of haemolysis assay indicated that the citric acid crosslinked carboxymethyl cellulose-tamarind gum hydrogels were safe to be used in drug delivery. These results indicated that the citric acid crosslinked carboxymethyl cellulose-tamarind gum composite hydrogel film has the potential to be used in topical novel drug delivery systems.
\end{abstract}

Key words: Carboxymethyl cellulose, citric acid, crosslinking, hydrogel, tamarind gum

Hydrogels are the crosslinked networks of hydrophilic water-soluble polymers. They have a tendency to absorb enormous water and swell[1]. Their distinct physical properties make them suitable for drug delivery applications. The hydrogels meant for drug delivery can be prepared using synthetic and natural polymers $^{[2-8]}$. However, natural polymer-based hydrogels are more preferable due to their inherent biocompatible and biodegradable nature ${ }^{[9]}$. Besides, the natural polymers are cheap as compared to the synthetic polymers. Some of the well-known natural polymers used for the preparation of hydrogels include cellulose $^{[10]}$, $\operatorname{starch}^{[11]}$, chitosan ${ }^{[12]}$, sodium alginate ${ }^{[13]}$, guar gum $^{[8]}$, gellan gum ${ }^{[14]}$ and xanthan gum ${ }^{[7]}$.

The natural polymer-based hydrogels can be prepared by physical or chemical crosslinking. The physically crosslinked hydrogels are mechanically weak and change in the environmental parameters such as $\mathrm{pH}$, temperature or ionic strength may disrupt the

*Address for correspondence E-mail: malikailas@gmail.com

July-August 2018 network structure of the hydrogels ${ }^{[15]}$. The chemically crosslinked hydrogels are strong but the crosslinking agents used for their preparation are reported to be toxic $^{[11]}$. In past few years, citric acid has emerged as a non-toxic crosslinking agent for the preparation of the hydrogels ${ }^{[16,17]}$. At sufficiently high temperatures, citric acid forms a cyclic anhydride and esterifies the hydroxyl groups present on the adjacent polymer chains. This leads to the formation of crosslinks.

Carboxymethylcellulose (CMC) is a biodegradable and easily available derivative of cellulose, which is considered as an ideal candidate for preparing hydrogels due to its high swellability. It has hydrophilic

This is an open access article distributed under the terms of the Creative Commons Attribution-NonCommercial-ShareAlike 3.0 License, which allows others to remix, tweak, and build upon the work non-commercially, as long as the author is credited and the new creations are licensed under the identical terms

Accepted 30 May 2018

Revised 30 October 2017

Received 11 March 2017

Indian J Pharm Sci 2018;80(4):657-667 
carboxylate groups in its polymeric backbone. When used alone, CMC forms hydrogels with poor mechanical strength due to formation of intramolecular crosslinks instead of intermolecular crosslinks. This can be attributed to the electrostatic repulsion between charged macromolecules due to which, few hydroxyl groups remain available for reaction at C6, the most reactive position. Therefore, another cellulose derivative like hydroxyethylcellulose (HEC) has been used in combination with $\mathrm{CMC}$ to improve the intermolecular crosslinking ${ }^{[18]}$.

Tamarind gum (TG) is a natural polysaccharide, which is used as a binder, gelling agent, thickener, emulsifier, suspending agent and a release modifier in different pharmaceutical formulations. It is also used as a stabilizer in food and pharmaceutical industries ${ }^{[19]}$. It is extracted from the endosperm of Tamarindus indica $\mathrm{L}$. seeds belonging to the family Leguminosae. TG is composed of $(1 \rightarrow 4)-\beta$-D-glucan back-bone substituted with side chains of $\alpha$-D-xylopyranose and $\beta$-D-galactopyranosyl $(1 \rightarrow 2)$ - $\alpha$-D-xylopyranose linked $(1 \rightarrow 6)$ to glucose residues ${ }^{[20]}$. It has been used in the development of various drug delivery systems due to its hydrophilic and bioadhesive properties ${ }^{[21-27]}$. Although TG shows presence of non-sterically hindered hydroxyl groups, very few reports mentioned the use of pure TG in chemically crosslinked hydrogels ${ }^{[28]}$. Moreover, TG can be used as a cheaper alternative to HEC for the preparation of citric acid crosslinked CMC-based hydrogels.

Moxifloxacin hydrochloride (MH) is a fourth generation fluoroquinolone, widely used for soft tissue and skin infections, which promoted wound healing without causing bacterial resistance ${ }^{[29]}$. MH-loaded hydrogel film could be useful in treating topical infections as well as burn wounds.

The objective of the present work was to investigate potential of citric acid crosslinked CMC-TG hydrogel films as topical drug delivery in the form of implants or wound healing films. In the present work, we have prepared the CMC-TG hydrogel films using citric acid as a crosslinking agent. The effect of concentration of TG on the properties of hydrogel films was investigated. MH was used as model drug. The hydrogel films were characterized for the formation of crosslinks and evaluated for swellability, drug loading and release. The biocompatibility of the hydrogel films was tested using haemolytic assay.

\section{MATERIALS AND METHODS}

Tamarind kernel powder was kindly gifted by Chhaya Industries, Barshi, Maharashtra, India. MH was obtained as a gift sample from Apotex, Bangalore, India. Sodium CMC (degree of substitution: 0.7, average molecular weight: 250000 ) and anhydrous citric acid anhydrous were procured from Sigma Aldrich, Mumbai, India. All other chemicals used were of analytical grade and were purchased from Loba Chemie, Mumbai, India.

\section{Isolation of TG:}

Twenty grams of defatted tamarind kernel powder was added to the cold distilled water $(200 \mathrm{ml})$ to prepare a slurry. The slurry was poured into the boiling distilled water $(800 \mathrm{ml})$ containing citric acid $(0.2 \%)$. The solution was boiled with stirring for $20 \mathrm{~min}$ on a water bath. The resulting thin clear solution was kept overnight $(24 \mathrm{~h})$ so that most of the proteins and fibres settle down, following which the solution was centrifuged at $5000 \mathrm{rpm}$ for $20 \mathrm{~min}$. The supernatant liquid was separated and poured into the excess of absolute alcohol with continuous stirring (1:1). The precipitated polysaccharide was washed with absolute ethanol, diethyl ether and acetone, followed by drying at $50-60^{\circ}$ for $10 \mathrm{~h}$. The dried polymer was powdered, sieved (sieve no: 80 ), and stored in a desiccator until further use ${ }^{[30]}$. Extracted TG was further qualitatively evaluated for the presence of proteins and fats.

\section{Preparation of citric acid crosslinked TG-based hydrogel films:}

The citric acid crosslinked hydrogel films of TGCMC were prepared by esterification-crosslinking mechanism, as reported elsewhere ${ }^{[10]}$. Two percent $\mathrm{w} / \mathrm{v}$ aqueous solutions containing $\mathrm{TG}, \mathrm{CMC}$ and citric acid were prepared using a mechanical stirrer (RQG126D, Remi, India), at room temperature (Table 1). The solutions were kept overnight to remove the entrapped air bubbles. The aqueous solutions $(50 \mathrm{ml})$ were casted into $9 \mathrm{~cm}$ diameter Petri dishes and

\section{TABLE 1: CMC AND TG HYDROGEL FILMS}

\begin{tabular}{lccccc}
\hline \multirow{2}{*}{ Parameter } & \multicolumn{5}{c}{ Batch code } \\
\cline { 2 - 6 } & B1 & B2 & B3 & C1 & D1 \\
\hline TG:CMC & $1: 1$ & $2: 1$ & $3: 1$ & $1: 1$ & $1: 1$ \\
Citric acid (\%) & 10 & 10 & 10 & 15 & 20 \\
Curing temperature $\left({ }^{\circ}\right)$ & 140 & 140 & 140 & 140 & 140 \\
Curing time (min) & 5 & 5 & 5 & 5 & 5 \\
\hline
\end{tabular}

TG- tamarind gum; CMC- carboxymethyl cellulose (sodium salt); *total polymer concentration equivalent to $2 \% \mathrm{w} / \mathrm{v}$ 
dried at $50^{\circ}$ for $24 \mathrm{~h}$. The resulting films were cured at $140^{\circ}$ for $5 \mathrm{~min}$. The curing temperature and curing time was sufficient to achieve crosslinking. The cured hydrogel films were washed with distilled water and isopropyl alcohol for $1 \mathrm{~h}$ in order to remove untreated entities. Then, the hydrogel films were dried in a hot air oven at $50^{\circ}$ for $24 \mathrm{~h}$ and stored in a desiccator until use. The parameters such as concentrations of TG, CMC, citric acid and curing time were varied individually to study their effect on hydrogel properties ${ }^{[31]}$.

\section{Total carboxyl content of hydrogel films:}

Acid-base titration was used to determine total carboxyl content of the hydrogel films. About $100 \mathrm{mg}$ of hydrogel film was dissolved in $20 \mathrm{ml} 0.1 \mathrm{~N} \mathrm{NaOH}$ and stirred on magnetic stirrer (Remi, India) for $2 \mathrm{~h}$. Excess amount of $0.1 \mathrm{~N} \mathrm{NaOH}$ was titrated with 0.1 $\mathrm{N} \mathrm{HCl}$ using phenolphthalein as an indicator ${ }^{[32]}$. The carboxyl content in milliequivalents per $100 \mathrm{~g}$ of hydrogel films was calculated as given below, carboxyl content $=\left(\mathrm{V}_{\mathrm{b}}-\mathrm{V}_{\mathrm{a}}\right) \times \mathrm{N} \times 100 / \mathrm{W}$, where, $\mathrm{N}$ is the normality of $\mathrm{HCl}$ (eq./1), $\mathrm{V}_{\mathrm{b}}$ and $\mathrm{V}_{\mathrm{a}}$ are the volumes of $\mathrm{HCl}$ in absence and presence of sample, and $\mathrm{W}$ is the weight of sample $(\mathrm{g})$.

\section{Attenuated total reflectance-Fourier transform infrared (ATR-FTIR) spectroscopy:}

Infrared spectra of TG, CMC, citric acid, and citric acid crosslinked hydrogel films were obtained using ATR-FTIR spectrophotometer (Shimadzu, Miracle 10, IR Affinity, Japan). The samples to be analysed were placed in the ATR and spectra were recorded in the range of $600-4000 \mathrm{~cm}^{-1}$ at an average of 25 scans and resolution of $4 \mathrm{~cm}^{-1}$.

\section{Solid-state NMR spectroscopy:}

Solid-state ${ }^{13} \mathrm{C}$ cross-polarization-magic angle spinning $\left({ }^{13} \mathrm{C}\right.$ CP-MAS) NMR spectra of TG, CMC and citric acid crosslinked hydrogel film was measured using Jeol-ECX400 spectrometer operating at $400 \mathrm{MHz}$ (contact time of $3.5 \mathrm{~ms}$, relaxation delay of $5 \mathrm{~s}$, sweep width of $35 \mathrm{kHz}$ and spinning speed of $10 \mathrm{KHz}$ ). The chemical shifts were calibrated with the external hexamethylbenzene standard methyl resonance at $17.3 \mathrm{ppm}$.

\section{Thermal analysis:}

Thermogravimetric analysis (TGA) and differential scanning calorimetry (DSC) of TG, CMC, citric acid and crosslinked hydrogel film was performed using a Mettler-Toledo TGA/DSC1 thermogravimetric analyser (Mettler-Toledo, Switzerland). Samples were heated from $30^{\circ}-300^{\circ}$ at a rate of $10^{\circ} / \mathrm{min}$, under nitrogen atmosphere (flow rate: $10 \mathrm{ml} / \mathrm{min}$ ).

\section{Swelling study:}

The extent of swelling of hydrogel films was determined in phosphate buffer $\mathrm{pH}$ 7.4. A known quantity $(\sim 0.2 \mathrm{~g})$ of hydrogel films were immersed in the swelling medium $(10 \mathrm{ml})$ at room temperature. The swollen samples were removed at predetermined time intervals for $24 \mathrm{~h}$, excess medium was blotted using tissue paper and the swollen hydrogel were weighed ${ }^{[33]}$. The swelling index was calculated using the following Eqn., swelling ratio $(\mathrm{g} / \mathrm{g})=\left(\mathrm{W}_{\mathrm{T}}-\mathrm{W}_{0}\right) / \mathrm{W}_{\mathrm{T}}$, where, $\mathrm{W}_{\mathrm{T}}$ is the weight of swollen hydrogel at time $\mathrm{T}$, and $\mathrm{W}_{0}$ is the weight of dry hydrogel films before start of the study. All measurements were done in triplicate.

\section{Drug loading and in vitro release:}

MH was loaded into hydrogel films using the swelling equilibrium method. In brief, pre-weighed hydrogel films ( $\sim 200 \mathrm{mg})$ were placed in $20 \mathrm{ml}$ aqueous solution of $\mathrm{MH}(5 \mathrm{mg} / \mathrm{ml})$ for $2 \mathrm{~h}$ and hydrogel films were dried in hot air oven at $40^{\circ}$ for $24 \mathrm{~h}^{[34]}$. To determine the amount of drug loading, MH-loaded hydrogel film was cut into small pieces, weighed and immersed in $50 \mathrm{ml}$ water. The dispersion was stirred on a magnetic stirrer (Remi, India) at $100 \mathrm{rpm}$ for $24 \mathrm{~h}$ and the amount of $\mathrm{MH}$ was determined spectrophotometrically using a $\mathrm{UV} /$ Vis spectrophotometer (Shimadzu, Japan) at $\lambda_{\max }$ $289 \mathrm{~nm}$.

In vitro release studies of the drug were carried out by keeping the MH-loaded hydrogel films $(\sim 0.1 \mathrm{~g})$ in $20 \mathrm{ml}$ of phosphate buffer $\mathrm{pH} 7.4$ at $37^{\circ}$. Samples were withdrawn at predetermined time intervals, each time replacing the volume withdrawn with fresh dissolution medium in order to maintain the sink conditions. The amount of $\mathrm{MH}$ released was determined using a UV/Vis spectrophotometer at $\lambda_{\text {max }} 289 \mathrm{~nm}$ up to $300 \mathrm{~min}$. The experiments were performed in triplicate $^{[35]}$.

\section{Haemolysis assay:}

Hydrogel films $\left(2 \mathrm{~cm}^{2}\right)$ were equilibrated in phosphatebuffered saline for $60 \mathrm{~min}$ at $37^{\circ}$ and human citratephosphate-dextrose (CPD) blood $(0.5 \mathrm{ml})$ was added to the films. After $20 \mathrm{~min}, 4.0 \mathrm{ml}$ of $0.9 \%$ sodium chloride solution (saline) was added to each sample to stop haemolysis and the samples were incubated for $60 \mathrm{~min}$ at $37^{\circ}$. Positive and negative controls were obtained by adding $0.5 \mathrm{ml}$ of human CPD blood and 
saline, respectively, to $4.0 \mathrm{ml}$ of double-distilled water. The incubated samples were centrifuged for $10 \mathrm{~min}$ at $3500 \mathrm{rpm}$, the supernatant was taken, and its absorbance was measured on a spectrophotometer at $545 \mathrm{~nm}^{[31]}$. Percent haemolysis was calculated using the following relationship: haemolysis $(\%)=\left(\mathrm{A}_{\text {Test sample }}-\mathrm{A}_{\text {-ve control }}\right) /$ $\left(\mathrm{A}_{+\mathrm{ve} \text { control }}-\mathrm{A}_{-\mathrm{ve} \mathrm{control}}\right) \times 100$, where, $\mathrm{A}$ is the absorbance. The absorbance of positive and negative controls was found to be 3.614 and $0.011 \%$, respectively.

\section{Statistical analysis:}

The numerical data was statistically analysed using one way ANOVA followed by Tukey's multiple comparison test. $\mathrm{P}$ value less than 0.05 was considered as statistically significant.

\section{RESULTS AND DISCUSSION}

TG was isolated from seeds of tamarind (T. indica), and the average yield of dried TG was found to be $48 \%$. The addition of citric acid helped to separate proteins from the TG due to precipitation. The TG present in the supernatant solution was separated by alcohol precipitation ${ }^{[36]}$. The molecular weight of TG is $1.5 \times 10^{5} \mathrm{~g} / \mathrm{mol}$. Extracted polysaccharide was free from proteins and fats.

The preformulation batches were prepared in order to optimize concentration of citric acid, curing temperature and curing time to get hydrogel film. The prepared hydrogel films were washed with distilled water to remove unreacted citric acid and polymer. The total polymer concentration was fixed at $2 \%$ and TG to $\mathrm{CMC}$ ratio was varied from $1: 1$ to $3: 1$. The average thickness of citric acid crosslinked TG/CMC hydrogel film was found to be $\sim 174 \mu \mathrm{m}$.

At very low concentrations of citric acid, the TGbased hydrogel films were not formed due to poor crosslinking, which may have led to loss of integrity of hydrogel film. At high concentrations of citric acid, the hydrogel films formed were rigid, with reduced ability to absorb water ${ }^{[37]}$. This rigidity and poor ability to absorb water might be due to the increase in crosslinking density, which in turn may have led to the reduction in the mobility of polymer chains and reduced the free volume of the hydrogel network ${ }^{[2]}$.

The curing temperature was varied from $130^{\circ}$ to $150^{\circ}$. At lower temperature poor crosslinked gel was formed, whereas at elevated temperature, firm and tough hydrogel film was formed. This might be due to the formation of strong hydrogen bond interactions between polysaccharides ( $\mathrm{TG}$ and $\mathrm{CMC}$ ) and citric acid, leading to reduction in expansion and relaxation of polymer chains ${ }^{[37]}$. The optimum swelling was observed at a curing temperature $140^{\circ}$.

The curing time was varied from 5 to $15 \mathrm{~min}$. The 5 min curing time was found to be sufficient to form the citric acid crosslinked hydrogel film while higher curing times lead to formation of a brown-colored film with insufficient swelling. This could be exhibited due to interaction between - $\mathrm{OH}$ groups of polysaccharide and dehydrated citric acid leading to the formation of strong crosslinking.

Esterification reaction caused the formation of crosslinks between citric acid and polysaccharides (TG and CMC). The possible reaction and structure of TG and CMC were given in fig. 1. When citric acid is heated, the cyclic anhydride intermediate is formed, which served as the base mechanism responsible for the development of crosslinks with $\mathrm{TG}$ and $\mathrm{CMC}$. Esterification of $-\mathrm{OH}$ functional groups of the polysaccharide with the cyclic anhydride intermediate lead to new carboxylic acid units, which exhibited the property of forming new intra-molecular anhydride moiety with neighbouring carboxylic acid unit ${ }^{[10,38,39]}$. There might also be involvement of the primary $-\mathrm{OH}$ groups of the polysaccharides as they are more reactive than the secondary $-\mathrm{OH}$ groups, in the esterification process. Crosslinking generally occurred under dry state and required high temperatures for the reaction ${ }^{[1]}$. On the other hand evaporation of water, which is the first step in crosslinking with citric acid, is promoted by the hydrophobic character of the substrate.

The carboxyl content of hydrogel films was found to be in the range of 2.59 to $4.35 \mathrm{mEq} / \mathrm{g}$ of hydrogel (Table 2). The ratio of polysaccharide and concentration of citric acid had significant effect on the carboxyl content. When TG was increased in the films (B1 to B3), the carboxyl content was found to be increased. Increase in carboxyl content might be due to increased participation of TG in the esterification reaction with citric acid. An increase in concentration of citric acid from 10 to $20 \%$ (B1, C1 and D1), also increased the carboxyl content of hydrogel film. This could be due to fact that when the film was heated, higher amount of cyclic anhydride intermediate of citric acid was available for crosslinking of polysaccharides leading to increased carboxyl content.

ATR-FTIR spectra of CMC, TG and citric acid were presented in fig. 2A and spectra of all hydrogel films 


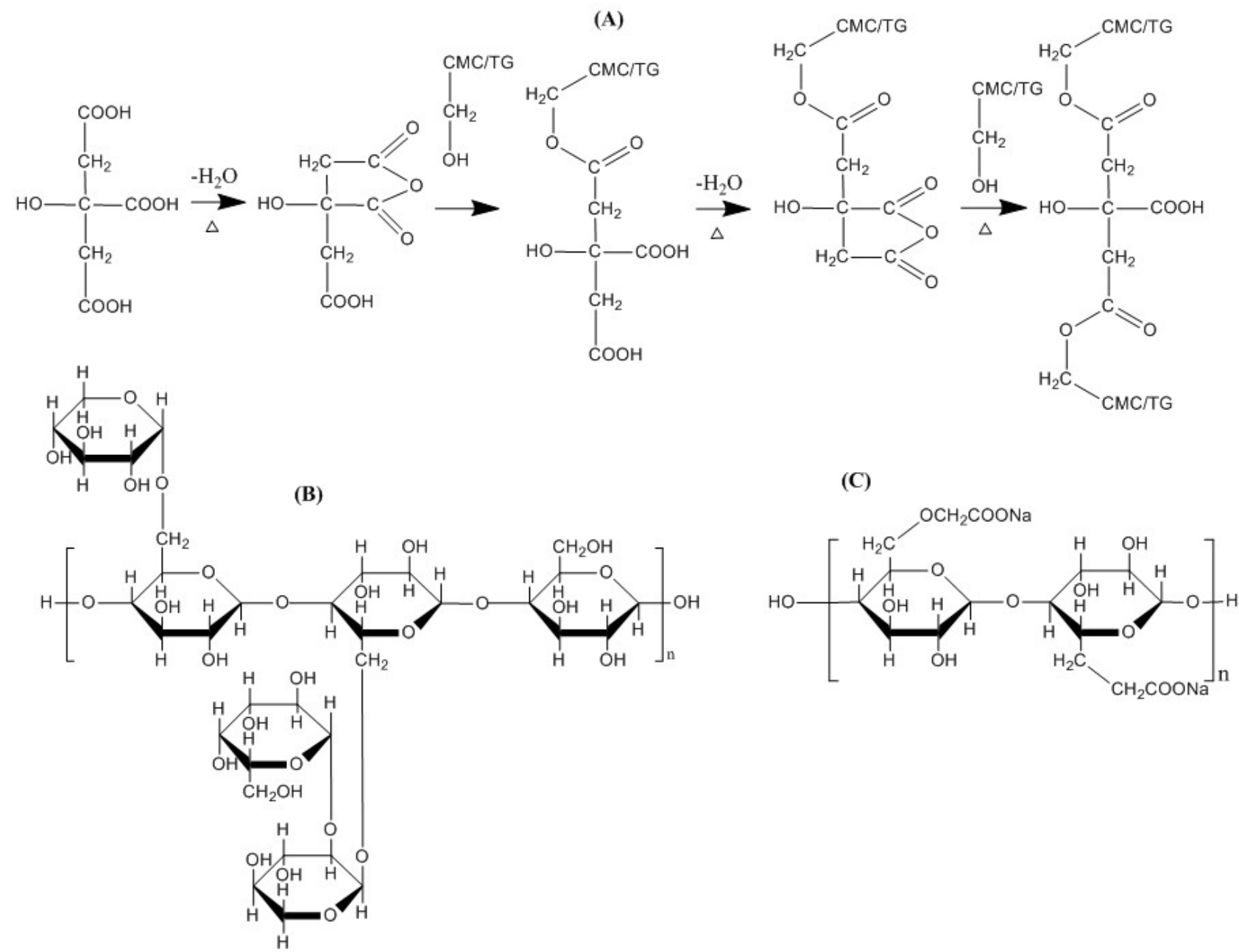

Fig. 1: Possible crosslinking reaction between citric acid, TG and CMC (A), structure of tamarind gum (B) and structure of carboxymethyl cellulose (C)

TABLE 2: CHARACTERIZATION OF HYDROGEL FILMS

\begin{tabular}{lcccccc}
\hline Batch & $\begin{array}{c}\text { Total carboxyl content } \\
(\mathrm{mEq} / \mathrm{g} \text { of hydrogel) }\end{array}$ & $\begin{array}{c}\text { Drug loading* } \\
(\mathbf{m g} / \mathbf{g})\end{array}$ & $\begin{array}{c}\text { Haemolysis } \\
(\%)\end{array}$ & $\mathbf{n}$ & $\mathbf{r}^{2}$ & Order of release \\
\hline B1 & 2.59 & $402.3 \pm 4.19$ & 1.56 & 0.60 & 0.972 & Quassi-Fickian \\
B2 & 3.22 & $249.1 \pm 5.12^{\mathrm{a}}$ & 1.14 & 0.75 & 0.991 & Non-Fickian \\
B3 & 3.34 & $129.8 \pm 4.51^{\mathrm{a}}$ & 2.57 & 0.86 & 0.990 & Non-Fickian \\
C1 & 3.38 & $268.6 \pm 3.41^{\mathrm{a}}$ & 1.79 & 0.75 & 0.993 & Non-Fickian \\
D1 & 4.08 & $256.8 \pm 2.69^{\mathrm{a}}$ & 1.93 & 0.76 & 0.994 & Non-Fickian \\
\hline
\end{tabular}

*Mean \pm standard deviation; ${ }^{a}$ significantly different at $p<0.05$ from B1; $n$-release exponent; $r^{2}$ - correlation coefficient

were given in fig. 2B. ATR-FTIR spectrum of TG exhibited broad strong peaks at $3500-3000 \mathrm{~cm}^{-1}$ due to stretching vibration of - $\mathrm{OH}$. A strong peak at 1039 and $1143 \mathrm{~cm}^{-1}$ could be attributed to the $\mathrm{C}-\mathrm{O}$ stretching vibration of the alcoholic group. The medium peak at $2920 \mathrm{~cm}^{-1}$ belonged to asymmetric stretching of $\mathrm{CH}$. The peaks at 1747 and $1689 \mathrm{~cm}^{-1}$ were due to carbonyl $(-\mathrm{HC}=\mathrm{O})$ stretching $^{[40]}$. The spectrum of citric acid showed a broad peak at $3279 \mathrm{~cm}^{-1}$ attributed to $-\mathrm{OH}$ stretching and a sharp peak at $1693 \mathrm{~cm}^{-1}$ due to hydrogen bonded $\mathrm{C}=\mathrm{O}$ stretch. In the spectrum of $\mathrm{CMC}$, the broad peaks at 3500 to $3000 \mathrm{~cm}^{-1}$ are seen due to $-\mathrm{OH}$ stretching. The band at $2922 \mathrm{~cm}^{-1}$ is due to $\mathrm{C}-\mathrm{H}$ stretching vibration. The presence of a strong absorption band at 1587 and $1411 \mathrm{~cm}^{-1}$ was due to the asymmetric and symmetric stretching of $\mathrm{COO}^{-}$group, respectively. The peak at $1323 \mathrm{~cm}^{-1}$ was due to $\mathrm{CH}-\mathrm{O}-$ $\mathrm{CH}_{2}$ stretching. The ATR-FTIR spectrum of hydrogel film showed additional peaks at 1710 to $1730 \mathrm{~cm}^{-1}$ attributed to the carbonyl band of free carboxylic acid groups and the carbonyl band of ester formed during the TG-CMC, CMC-CMC and TG-TG crosslinking. To confirm the formation of ester linkages further, hydrogel film was treated with $0.1 \mathrm{~N} \mathrm{NaOH}$ in 
methanol so as to separate ester carbonyl band from the acid carbonyl band by converting the carboxylic acid group to carboxylate anions. In case of $\mathrm{NaOH}$-treated batch (fig. 1A) additional two bands were observed at $1742 \mathrm{~cm}^{-1}$ (ester carbonyl) and $1589 \mathrm{~cm}^{-1}$ (carboxylate), which confirmed crosslinking ${ }^{[31]}$. Solid-state ${ }^{13} \mathrm{C}$ NMR spectrum of TG showed three distinct peaks (fig. 3). The resonance peak at $105 \mathrm{ppm}$ is assigned

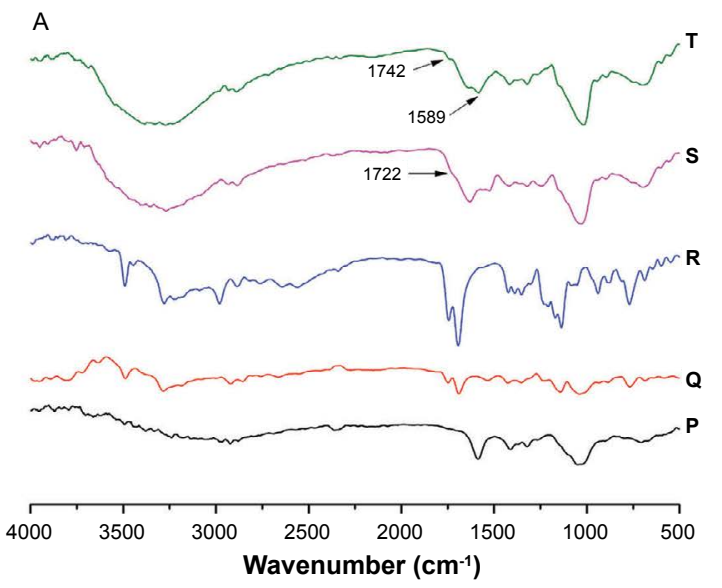

to anomeric carbon atom $(\mathrm{C} 1)$ and the peak at 74 ppm is assigned to the carbon atoms ( 2 2 to $\mathrm{C} 5$ ) connected by $-\mathrm{OH}$ groups (i.e. the carbon atoms in the six membered ring except $\mathrm{C} 1$ carbon atom). The presence of a peak at $63 \mathrm{ppm}$ is attributed to the C6 carbon atom of $\mathrm{CH}_{2} \mathrm{OH}$ group. The solid-state ${ }^{13} \mathrm{C}$ NMR spectrum of CMC showed four distinct peaks (fig. 3). The resonance peak at $105 \mathrm{ppm}$ is attributed

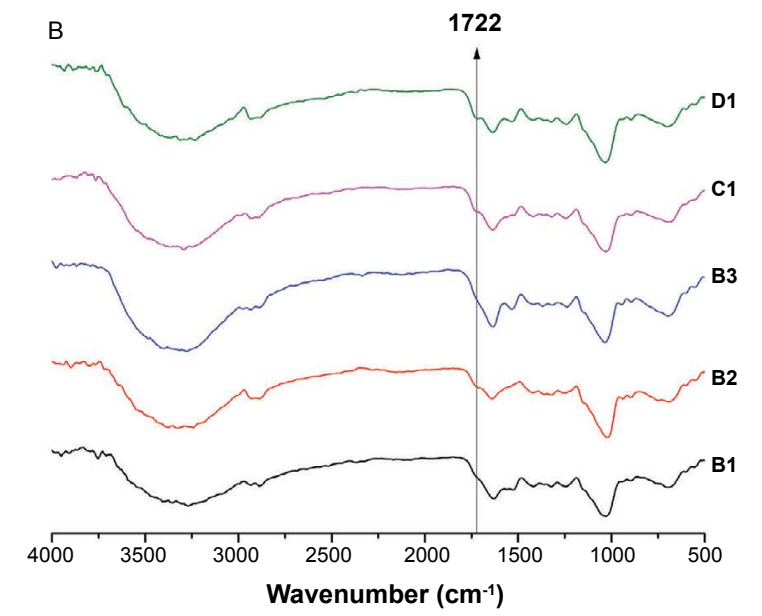

Fig. 2: ATR-FTIR spectra

(A) ATR-FTIR spectra of P. carboxymethyl cellulose, Q. tamarind gum, R. citric acid, S. hydrogel film and T. NaOH-treated hydrogel film; (B) batches of hydrogel films B1, B2, B3, C1 and D1.

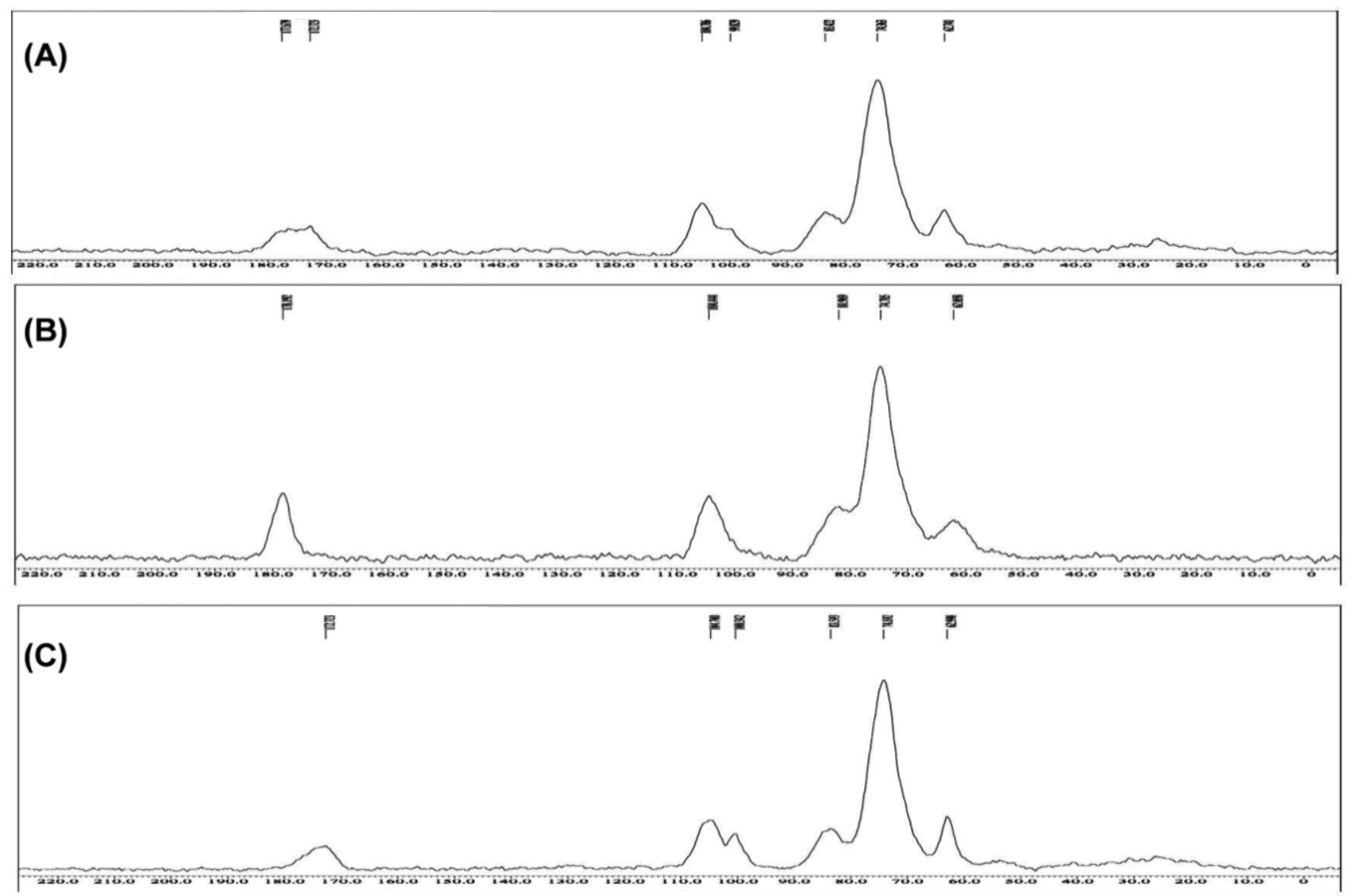

Fig. 3: Solid state ${ }^{13} \mathrm{C}$ NMR spectra

Solid-state ${ }^{13} \mathrm{C}$ NMR spectra of A. hydrogel film, B. carboxymethyl cellulose and C. tamarind gum 
to anomeric carbon $(\mathrm{C} 1)$ of glucose units and the peak at $75 \mathrm{ppm}$ is due to the carbon atoms ( $\mathrm{C} 2$ to $\mathrm{C} 5)$ in the six member ring connected by $-\mathrm{OH}$ groups and C7 carbon atom in methyl group of carboxylated side chain. The presence of a peak at $63 \mathrm{ppm}$ is attributed to $\mathrm{C} 6$ of the carbon atom of $\mathrm{CH}_{2} \mathrm{O}$ - group. The peak at $178 \mathrm{ppm}$ is due to carbonyl carbon of substituent groups $-\mathrm{CH}_{2} \mathrm{COO}^{-}$. The solid-state ${ }^{13} \mathrm{C}$ NMR of prepared citric acid crosslinked hydrogel film (fig. 3) of TG-CMC shows broad resonance peak due to ester crosslinks and free $-\mathrm{COOH}$ groups in the range of 174-182 ppm, which confirms crosslinking.

The TGA and DSC thermograms were presented in the fig. 4A and 4B, respectively. Thermal decomposition curve of TG showed two main stages of decomposition. The first stage began at $35^{\circ}$ and ended at $100^{\circ}$. This could be due to the removal of free and bound water from the polymer. The second stage of weight loss was observed around $228^{\circ}$ to $300^{\circ}$ with $35 \%$ loss of weight. CMC showed two main stages of decomposition. The first stage began at $35^{\circ}$ and ended at $84^{\circ}$ and was due to removal of free and bound water from the polymer. The second stage of weight loss was observed around $225^{\circ}$ to $325^{\circ}$ with $38 \%$ loss of weight. The weight loss in both the polymers in second stage could be attributed to the decomposition of the polymer backbone. The decomposition of anhydrous citric acid started at $160^{\circ}$ and ended at $349^{\circ}$ with $93 \%$ weight loss. In case of hydrogel film, thermal decomposition started at $199^{\circ}$ and ended at $389^{\circ}$ with $47.5 \%$ loss of weight. This indicated that the decomposition of the citric acid crosslinked TG/CMC hydrogels is slower than individual polymers. The thermal stability of

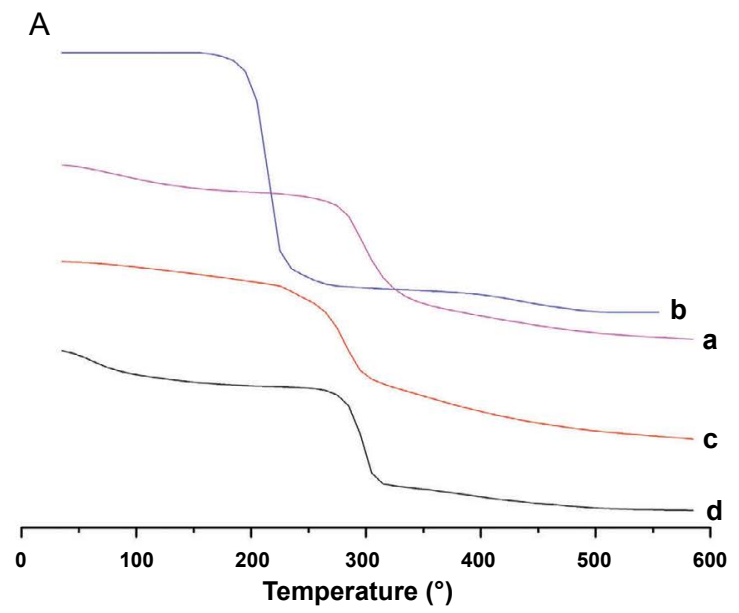

the prepared hydrogel film was improved compared to that of the individual polymers due to formation of crosslinks between TG/CMC by citric acid. In other words, thermal decomposition of TG and CMC was delayed. The DSC thermogram of hydrogel film showed considerable change in the heat flow indicating formation of ester crosslinks. Observations are in agreement with the earlier reports with $\mathrm{CMC}$ and hydroxyethyl cellulose ${ }^{[10,41]}$.

The swelling study was performed in phosphate buffer $\mathrm{pH}$ 7.4. The result of swelling study presented in fig. 5A. Batch B1 exhibited a maximum equilibrium swelling of $5.33 \mathrm{~g} / \mathrm{g}$ whereas batch D1 showed a minimum equilibrium swelling of $1.64 \mathrm{~g} / \mathrm{g}$. When hydrogel is in contact with medium, its three dimensional hydrophilic network expansion is prevented by the crosslinks and provided an elastic response, which affected the swelling of hydrogel film ${ }^{[42]}$. All hydrogel batches showed steady swelling ratio after $1 \mathrm{~h}$.

The swelling ratio of the hydrogel film at equilibrium is dependent on the ratio of polymer and the concentration of citric acid. As the polymer ratio was increased the equilibrium swelling ratio was found to be decreased significantly (batch B1 to B3). This might be attributed to high amount of $\mathrm{TG}$ and low amount of $\mathrm{CMC}$ in hydrogel film, which decreased ionic character and increased hydrophilicity of hydrogel film ${ }^{[43]}$. In case of batch B3 there might be self-entanglement of TG chains by hydrogen bonding and decreased ionic contribution to the absorption of swelling medium leading to decreased equilibrium swelling ${ }^{[44]}$. In case of batch B1 there might be dissociation of highly hydrophilic carboxylic group of CMC, which absorbed a lot of

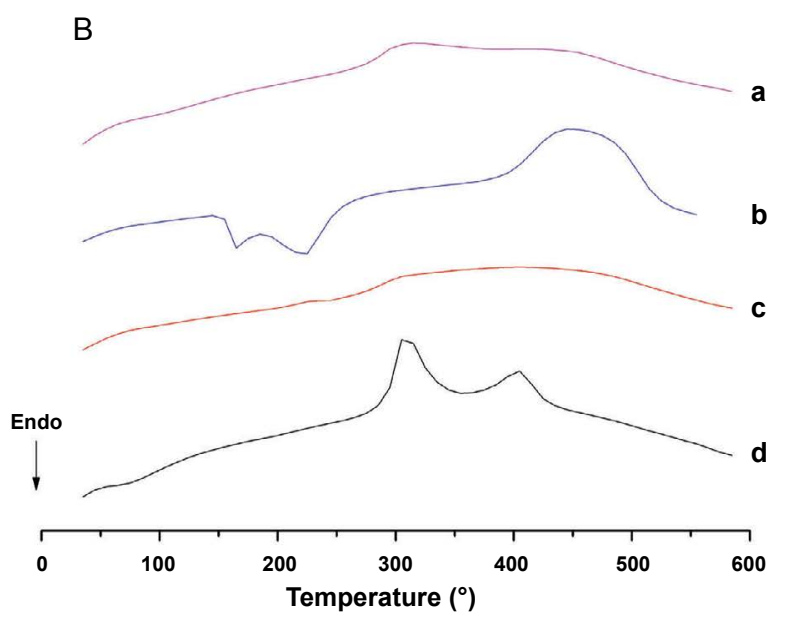

Fig. 4: TGA and DSC thermograms

A. TGA thermograms and B. DSC thermograms of a. hydrogel film, b. citric acid, c. TG and d. CMC 


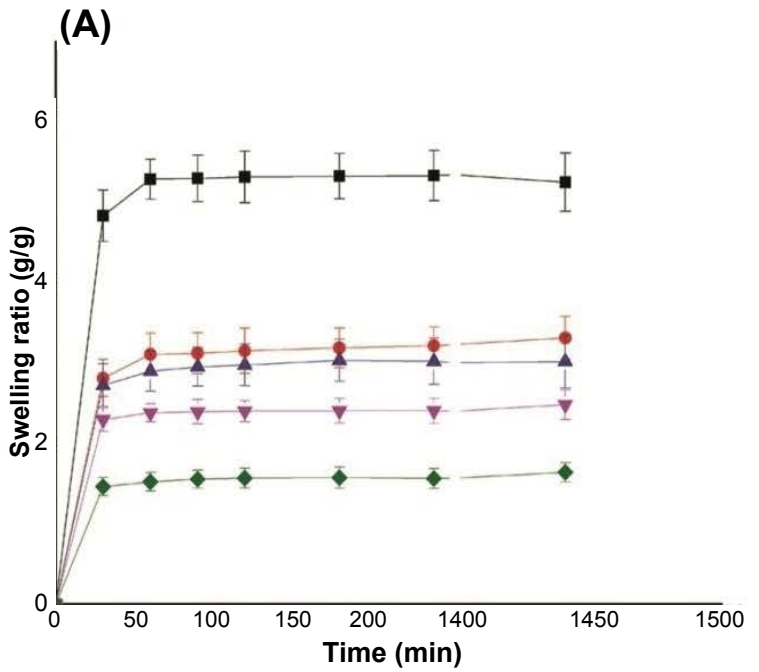

Fig. 5: Swelling ratio of and drug release from hydrogel films A. Swelling rations and $\mathrm{B}$. \% drug release of hydrogels $\mathrm{B} 1-\square-$

water to fill pores leading to increased equilibrium swelling ${ }^{[45,46]}$.

It was noticed that when concentration of citric acid was increased the swelling ratio of hydrogel film was found to be decreased significantly (fig. 4A). This might be due to an increase in the degree of crosslinking, which altered the mobility of polymer chains. Diffusion of the swelling medium into the polymer network also decreased thereby giving rise to more rigid structure of the polymer network ${ }^{[37]}$. As discussed earlier the amount of carboxyl content in hydrogel increased with increase in extent of cross-linking density.

The drug loading was done in aqueous solution of $\mathrm{MH}$ through diffusion process in swollen networks. The results of drug loading studies was presented in Table 2. Drug loading of hydrogel film was observed in the range of 402.3 to $84.78 \mathrm{mg} / \mathrm{g}$ of hydrogel film. As the degree of equilibrium swelling increased, drug loading was found to be increased. This might be due to faster diffusion of drug in to swollen network. Drug loading was found to be decreased as the polymerweight ratio and concentration of crosslinker citric acid was increased. This is probably due to low equilibrium swelling ratio of the hydrogel films.

As the prepared hydrogel films could be used to deliver the drug topically or as implants, in vitro drug release study was performed at $\mathrm{pH} 7.4$. The in vitro drug release profile from $\mathrm{MH}$-loaded hydrogel films is given in fig. 5B. An initial burst of $\sim 15$ to $45 \%$ release of $\mathrm{MH}$ was observed from all CMC-TG hydrogel films. This could be due to release of the drug associated with the gel surface. The free drug molecules back diffused from

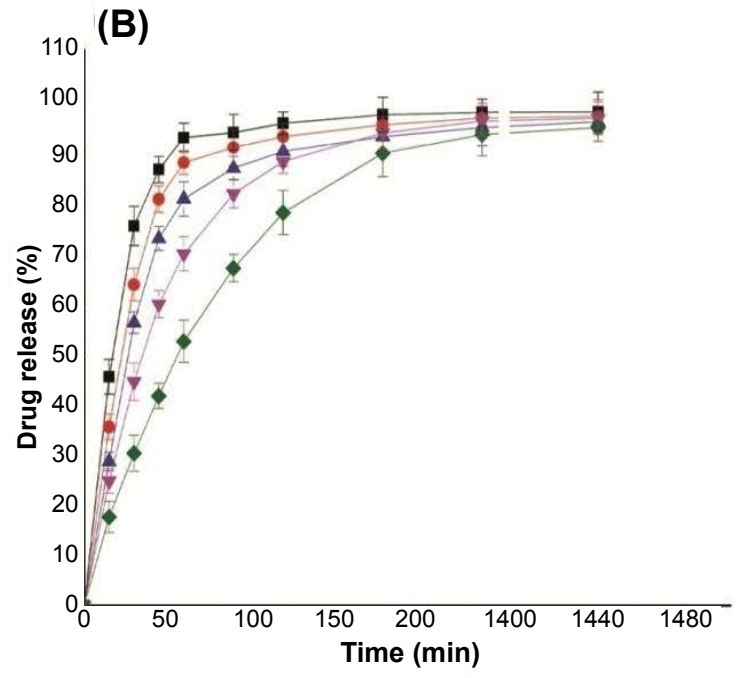

$\mathrm{B} 2-\bullet-, \mathrm{B} 3-\Delta-, \mathrm{C} 1-\nabla-$ and D1 $-\bullet$

the bulk of the hydrogel matrix at the surface along with solvent during drying of drug-loaded swollen hydrogel films. So, when the MH-loaded hydrogel film came in contact with the dissolution medium, the surface-associated free drug is released at a faster rate. The controlled release was observed after $30 \mathrm{~min}$ from all drug-loaded hydrogel batches. The retardation of drug release is associated with the swelling of the drug-loaded hydrogel film. The swelling of hydrogel film increased the thickness of film from which drug gets diffused in to bulk of dissolution medium ${ }^{[34]}$.

High drug release was exhibited for batches B1 to B3. The equilibrium swelling of these batches was more than the other batches (C1 and D1) as evident from swelling study. Irrespective of the path length drug was released at a faster rate. This may be due to ionic contribution of the $\mathrm{CMC}$ at buffer $\mathrm{pH} 7.4$ as well as the low crosslinking of the hydrogels films. $\mathrm{MH}$ showed greater solubility above $\mathrm{pH} 6$ due to ionization of carboxylate group ( $\mathrm{pKa}=6.4)^{[47]}$. This could be one of the reasons why drug released at a faster rate form these hydrogel films. Drug release was found to be decreased in the hydrogel films from batch B1, C1 and D1, when the concentration of crosslinking agent was increased (fig. 4B). This might be due to the fact that as the concentration of citric acid was increased, the crosslinking density was also increased, which in turn decreased the swelling of the polymer matrix resulting in retardation of drug release.

The release data up to $60 \%$ of total drug released was fitted into the Korsmeyer-Peppas Eqn. to determine the release mechanism ${ }^{[31,48]} . M_{t} / M_{\infty}=a^{n}$, where $M_{t} / M_{\infty}$ is 
the fraction of drug released in time ' $t$ ', ' $a$ ' is the kinetic constant and ' $n$ ' is the diffusional coefficient, which depended upon the interaction in between drug and the components of hydrogel matrix. The result of diffusion coefficient (n) and release mechanism is given in Table 2. All hydrogel batches showed non-Fickian release behaviour. The value of diffusion coefficient was found to be greater than 0.5 indicating high interaction between the drug and the hydrogel and the drug was released by diffusion coupled with erosion mechanism. An increase in ' $n$ ' value from B1 to B3 suggested an increase in drug hydrogel interaction due to TG. Also, an increase in concentration of crosslinker (B1, C1, D1) increased the ' $n$ ' value.

The biocompatibility of the hydrogel films was determined by hemocompatibility study. The test is based on the determination of the lysis of red blood cells in presence of the hydrogel films. The released hemoglobin dissolved in external fluid giving a yellowish colour, which could be measured spectrophotometrically. The higher the optical density of the supernatant, the greater the cell damage ${ }^{[28]}$. The results of haemolysis assay of hydrogel films were given in Table 2. Percent haemolysis for all hydrogel films was found to be in the range of 1.14 to $2.79 \%$. Lower percent haemolysis for hydrogel film could be attributed to higher hydrophilicity of polymer matrix, which decreased polymer-RBC interactions and lowered disruption of $\mathrm{RBCs}^{[35]}$. The observed percent haemolysis was found to be less than the permissible limit of $5 \%{ }^{[49]}$ indicating hemocompatibility of hydrogel films.

CMC-TG hydrogel films were successfully developed using citric acid as the crosslinking agent. ATR-FTIR, TGA, DSC and solid-state ${ }^{13} \mathrm{C}$ NMR study indicated the formation of ester linkage between polymers. These results indicated that as the concentration of citric acid increased carboxyl content was increased and equilibrium swelling ratio found to be decreased. Citric acid-crosslinked CMC-TG composite hydrogel films exhibited high drug loading with controlled release of model drug. Haemolysis study indicated hemocompatibility of hydrogel films. It can be concluded that CMC-TG composite hydrogel films crosslinked with citric acid exhibit the potential to be used as controlled or sustained drug delivery system for topical delivery. Further, cytocompatibility and in vivo studies are required to further develop the CMCTG hydrogels as an approvable drug delivery system.

\section{Acknowledgements:}

Authors thank the President of YSPM's Yashoda Technical Campus, Satara, for providing necessary facilities for carrying out the research work. They acknowledge Shivaji University, Kolhapur and NMR facility centre of Indian Institute of Science, Bangalore for the assistance offered with analytical work. Also, authors wish to thank Apotex, Bangalore for providing gift sample of $\mathrm{MH}$.

\section{Conflicts of interest:}

The authors have no conflict of interest.

\section{Financial support and sponsorship:}

Nil.

\section{REFERENCES}

1. Peppas NA, Bures P, Leobandung W, Ichikawa H. Hydrogels in pharmaceutical formulations. Eur $\mathrm{J}$ Pharm Biopharm 2000;50(1):27-46.

2. Singh B, Sharma S, Dhiman A. Design of antibiotic containing hydrogel wound dressings: Biomedical properties and histological study of wound healing. Int $\mathrm{J}$ Pharm 2013;457(1):82-91.

3. Byun H, Hong B, Yong Nam S, Young Jung S, Won Rhim J, Bong Lee S. Swelling Behavior and Drug Release of Poly(vinyl alcohol) Hydrogel Cross-Linked with Poly(acrylic acid). Macromol Res 2008;16(3):189-93.

4. Pal K, Banthia AK, Majumdar DK. Preparation and characterization of polyvinyl alcohol-gelatin hydrogel membranes for biomedical applications. AAPS PharmSciTech 2007;8(1):21.

5. Bhattarai N, Gunn J, Zhang M. Chitosan-based hydrogels for controlled, localized drug delivery. Adv Drug Deliv Rev 2010;62(1):83-99.

6. Saarai A, Kasparkova V, Sedlacek T, Saha P. On the development and characterisation of crosslinked sodium alginate/gelatin hydrogels. J Mech Behav Biomed Mater 2013;18:152-66.

7. Shalviri A, Liu Q, Abdekhodaie MJ, Wu XY. Novel modified starch-xanthan gum hydrogels for controlled drug delivery: Synthesis and characterization. Carbohydr Polym 2010;79(4):898-907.

8. Das S, Subuddhi U. pH-Responsive guar gum hydrogels for controlled delivery of dexamethasone to the intestine. Int $\mathrm{J}$ Biol Macromol 2015;79:856-63.

9. Vasquez JMG, Tumolva TP. Synthesis and characterization of a self-assembling hydrogel from water-soluble cellulose derivatives and sodium hydroxide / thiourea solution. Am J Chem 2015;5(2):60-5.

10. Demitri C, Del Sole R, Scalera F, Sannino A, Vasapollo G, Maffezzoli A, et al. Novel superabsorbent cellulose-based hydrogels crosslinked with citric acid. J Appl Polym Sci 2008;110(4):2453-60.

11. Uliniuc A, Hamaide T, Popa M, Bacaita S. Modified Starch- 
Based Hydrogels Cross-Linked with Citric Acid and their use as Drug Delivery Systems for Levofloxacin. Soft Mater 2013;11(4):483-93.

12. Vaghani SS, Patel MM, Satish CS. Synthesis and characterization of $\mathrm{pH}$-sensitive hydrogel composed of carboxymethyl chitosan for colon targeted delivery of ornidazole. Carbohydr Res 2012;347(1):76-82.

13. Prabu D, Majdalawieh AF, Abu-Yousef IA, Inbasekaran $\mathrm{K}$, Balasubramaniam T, Nallaperumal N, et al. Preparation and characterization of gatifloxacin-loaded sodium alginate hydrogel membranes supplemented with hydroxypropyl methylcellulose and hydroxypropyl cellulose polymers for wound dressing. Int J Pharm Investig 2016;6(2):86-95.

14. Dhar S, Murawala P, Shiras A, Pokharkar V, Prasad BL. Gellan gum capped silver nanoparticle dispersions and hydrogels: cytotoxicity and in vitro diffusion studies. Nanoscale 2012;4(2):563-7.

15. Ahmed EM. Hydrogel: Preparation, characterization, and applications: A review. J Adv Res 2015;6(2):105-21.

16. Franklin DS, Guhanathan S. Synthesis and characterization of citric acid-based $\mathrm{pH}$-sensitive biopolymeric hydrogels. Polym Bull 2014;71(1):93-110.

17. Guimarães LF, Fidalgo TK, Menezes GC, Primo LG, Costa e Silva-Filho F. Effects of citric acid on cultured human osteoblastic cells. Oral Surg Oral Med Oral Pathol Oral Radiol Endod 2010;110(5):665-9.

18. Capitani D, Del Nobile MA, Mensitieri G, Sannino A, Segre AL. 13C solid-state NMR determination of crosslinking degree in super absorbing cellulose-based networks. Macromolecules 2000;33(2):430-7.

19. Pal D, Nayak AK. Novel tamarind seed polysaccharide-aginate mucoadhesive microspheres for oral gliclazide delivery: in vitro-in vivo evaluation. Drug Deliv 2012;19(3):123-31.

20. Jana S, Saha A, Nayak AK, Sen KK, Basu SK. Aceclofenacloaded chitosan-tamarind seed polysaccharide interpenetrating polymeric network microparticles. Colloids Surfaces B Biointerfaces 2013;105:303-9.

21. Datta R, Bandyopadhyay AK. A new nasal drug delivery system for diazepam using natural mucoadhesive polysaccharide obtained from tamarind seeds. Saudi Pharm J 2006;14(2):115-9.

22. Mali KK, Dhawale SC, Dias R, Havaldar V, Ghorpade V, Salunkhe N. Nasal Mucoadhesive In situ Gel of Granisetron Hydrochloride using Natural Polymers. J Appl Pharm Sci 2015;5(7):084-093.

23. Mali KK. Design and Optimization of Modified Tamarind Gum-based Floating-bioadhesive Tablets of Verapamil Hydrochloride. Asian J Pharm 2016;10(4):2-8.

24. Nayak AK, Pal D, Santra K. Development of calcium pectinatetamarind seed polysaccharide mucoadhesive beads containing metformin HCl. Carbohydr Polym 2014;101(1):220-30.

25. Ghelardi E, Tavanti A, Davini P, Celandroni F, Salvetti S, Parisio E, et al. A mucoadhesive polymer extracted from tamarind seed improves the intraocular penetration and efficacy of rufloxacin in topical treatment of experimental bacterial keratitis. Antimicrob Agents Chemother 2004;48(9):3396-401.

26. Mali KK, Dhawale SC, Dias RJ. Microemulsion Based Bioadhesive Gel of Itraconazole Using Tamarind Gum: In vitro and Ex vivo Evaluation. Marmara Pharm J 2017;21(3):688-700.

27. Mali KK, Dhawale SC, Dias RJ, Havaldar VD, Kavitake PR.
Interpenetrating networks of carboxymethyl tamarind gum and chitosan for sustained delivery of aceclofenac. Marmara Pharm J 2017;21(4):771-82.

28. Shaw GS, Uvanesh K, Gautham SN, Singh V, Pramanik K, Banerjee I, et al. Development and characterization of gelatintamarind gum/carboxymethyl tamarind gum based phaseseparated hydrogels: a comparative study. Des Monomers Polym 2015;18(5):434-50.

29. Jacobsen F, Fisahn C, Sorkin M, Thiele I, Hirsch T, Stricker I, et al. Efficacy of topically delivered moxifloxacin against wound infection by Pseudomonas aeruginosa and methicillinresistant Staphylococcus aureus. Antimicrob Agents Chemother 2011;55(5):2325-34.

30. Sumathi S, Ray AR. Release behaviour of drugs from tamarind seed polysaccharide tablets. J Pharm Pharm Sci 2002;5(1):12-8.

31. Ghorpade VS, Yadav AV, Dias RJ. Citric acid crosslinked cyclodextrin/hydroxypropylmethyl cellulose hydrogel films for hydrophobic drug delivery. Int J Biol Macromol 2016;93:75-86.

32. Ghorpade VS, Yadav AV, Dias RJ. Citric acid crosslinked $\beta$ -cyclodextrin/carboxymethyl cellulose hydrogel films for controlled delivery of poorly soluble drugs. Carbohydr Polym 2017;164:339-48.

33. Chen J, Park K. Synthesis and characterization of superporous hydrogel composites. J Control Release 2000;65(1-2):73-82.

34. Mali KK, Dhawale SC, Dias RJ. Synthesis and characterization of hydrogel films of carboxymethyl tamarind gum using citric acid. Int J Biol Macromol 2017;105:463-70.

35. Singh B, Dhiman A. Designing bio-mimetic moxifloxacin loaded hydrogel wound dressing to improve antioxidant and pharmacology properties. RSC Adv 2015;5(55):44666-78.

36. Rao P, Srivastava H. Tamarind. In: Whistler R, BeMiller J, editors. Industrial Gums Polysaccharides and Their Derivatives. 2nd ed. New York: Academic Press; 1973. p. 369-411.

37. Hashem M, Sharaf S, Abd El-Hady MM, Hebeish A. Synthesis and characterization of novel carboxymethylcellulose hydrogels and carboxymethylcellulolse-hydrogel-ZnOnanocomposites. Carbohydr Polym 2013;95(1):421-7.

38. Zhou YJ, Luner P, Caluwe P. Mechanism of crosslinking of papers with polyfunctional carboxylic acids. J Appl Polym Sci 1995;58(9):1523-34.

39. Xie X, Liu Q. Development and physicochemical characterization of new resistant citrate starch from different corn starches. Starch 2004;56(8):364-70.

40. Kulkarni RV, Mutalik S, Mangond BS, Nayak UY. Novel interpenetrated polymer network microbeads of natural polysaccharides for modified release of water soluble drug: In vitro and in vivo evaluation. J Pharm Pharmacol 2012;64(4):530-40.

41. Seki Y, Altinisik A, Demircioğlu B, Tetik C. Carboxymethylcellulose (CMC)-hydroxyethylcellulose (HEC) based hydrogels: Synthesis and characterization. Cellulose 2014;21(3):1689-98.

42. Sannino A, Maffezzoli A, Nicolais L. Introduction of molecular spacers between the crosslinks of a cellulose-based superabsorbent hydrogel: Effects on the equilibrium sorption properties. J Appl Polym Sci 2003;90(1):168-74.

43. Rathna GVN, Rao DVM, Chatterji PR. Hydrogels of GelatinSodium Carboxymethyl Cellulose: Synthesis and Swelling Kinetics. J Macromol Sci Part A 1996;33(9):1199-207.

44. Mishra A, Malhotra AV. Tamarind xyloglucan: a 
polysaccharide with versatile application potential. J Mater Chem 2009;19(45):8528.

45. Tang H, Chen H, Duan B, Lu A, Zhang L. Swelling behaviors of superabsorbent chitin/carboxymethylcellulose hydrogels. J Mater Sci 2014;49(5):2235-42.

46. Sannino A, Nicolais L. Concurrent effect of microporosity and chemical structure on the equilibrium sorption properties of cellulose-based hydrogels. Polymer 2005;46(13):4676-85.

47. Al Omari MMH, Jaafari DS, Al-Sou'od KA, Badwan AA.
Moxifloxacin hydrochloride. In: Profiles of drug substances, excipients, and related methodology. Burlington: Academic Press; 2014. p. 299-431.

48. Costa P, Sousa Lobo JM. Modeling and comparison of dissolution profiles. Eur J Pharm Sci 2001;13(2):123-33.

49. Dawlee S, Sugandhi A, Balakrishnan B, Labarre D, Jayakrishnan A. Oxidized chondroitin sulfate-cross-linked gelatin matrixes: Anew class of hydrogels. Biomacromolecules 2005;6(4):2040-8. 